\title{
Effect of Helicobacter pylori eradication on chronic gastritis during omeprazole therapy
}

B E Schenk, E J Kuipers, G F Nelis, E Bloemena, J C Thijs, P Snel, A E G Luckers, E C Klinkenberg-Knol, H P M Festen, P P Viergever, J Lindeman, S G M Meuwissen

Background-We have previously observed that profound acid suppressive therapy in Helicobacter pylori positive patients with gastro-oesophageal reflux disease is associated with increased corpus inflammation and accelerated development of atrophic gastritis.

Department of Gastroenterology, Sophia Hospital Zwolle, Netherlands G F Nelis

Department of Gastroenterology, Bethesda Hospital

Hoogeveen, Netherlands

J C Thijs

Department of Gastroenterology, Slotervaart Hospital Amsterdam,

Netherlands P Snel

Department of Gastroenterology, Maas Hospital Boxmeer, Netherlands A E G Luckers

Department of Gastroenterology, Groot Ziekengasthuis

Den Bosch, Netherlands

H P M Festen

Department of Gastroenterology, Gemini Hospital Den Helder, Netherlands P P Viergever

Department of Pathology, Free University Hospital Amsterdam,

Netherlands

E Bloemena

$\mathrm{J}$ Lindeman

Correspondence to: Dr B E Schenk, Department of Gastroenterology, Free University Hospital, PO Box 7057, 1007 MB Amsterdam, Netherlands \section{histological changes.}

Patients/methods-In a prospective randomised case control study, patients with reflux oesophagitis were treated with omeprazole $40 \mathrm{mg}$ once daily for 12 months. $H$ pylori positive patients were randomised to additional double blind treatment with omeprazole $20 \mathrm{mg}$, amoxicillin $1000 \mathrm{mg}$ and clarithromycin $500 \mathrm{mg}$ twice daily or placebo for one week. Biopsy sampling for histology, scored according to the updated Sydney classification, and culture were performed at baseline, and at three and 12 months. Results-In the persistently $H$ pylori positive group $(n=24)$, active inflammation increased in the corpus and decreased in the antrum during therapy $(p=0.032$ and $\mathbf{p}=0.002$, respectively). In contrast, in the $H$ pylori positive group that became $H$ pylori negative as a result of treatment $(n=33)$, active and chronic inflammation in both the corpus and antrum decreased $(p \leqslant 0.0001)$. The decrease in active and chronic inflammation in the corpus differed significantly compared with the persistently $H$ pylori positive group (both $\mathbf{p}=\mathbf{0 . 0 0 1}$ ). For atrophy scores, no significant differences were observed between $H$ pylori eradicated and persistently $H$ pylori positive patients within one year of follow up. No changes were observed in the $H$ pylori negative control group $(\mathrm{n}=26)$.

Conclusions-H pylori eradication prevents the increase in corpus gastritis associated with profound acid suppressive therapy. Longer follow up is needed to determine if $\boldsymbol{H}$ pylori eradication prevents the development of atrophic gastritis. (Gut 2000;46:615-621)

Keywords: Helicobacter pylori; gastritis; omeprazole; atropy; gastro-oesophageal reflux disease

Helicobacter pylori colonisation of the stomach almost always leads to inflammation of the mucosa. ${ }^{1-3}$ This chronic inflammation persists throughout life and may lead to gland loss and intestinal metaplasia. ${ }^{4-6}$ In some individuals this process proceeds to gastric cancer. Hence $H$ pylori associated gastritis is categorised as a distinct risk factor for the development of adenocarcinoma of the distal stomach. ${ }^{7-9}$ The mechanisms responsible for development of cancer have not been clarified in detail but the rate of progression to atrophy is related to the level of mucosal inflammation. The distribution and severity of gastritis depend on both the characteristics of the colonising $H$ pylori strain and the amount of acid production. If acid production is normal to high, bacterial colonisation and associated gastritis predominate in the antrum. If acid secretion is diminished, an equal pan-gastric or predominantly corpus gastritis pattern evolves in which the number of bacteria decreases in the antrum. The number of bacteria remains unchanged in the corpus but bacteria move in closer contact to the mucosa and into the gastric pits. ${ }^{6}{ }^{10} 11$ This pattern is reversible if acid secretion is restored. ${ }^{12}$

It is thought that the increase in antral $\mathrm{pH}$ impairs local growth conditions for $H$ pylori, perhaps as a result of its high urease activity. This redistribution of $H$ pylori and associated gastritis may have histopathological consequences. In various conditions associated with decreased gastric acid output, such as gastric ulcer disease or following vagotomy, accelerated gland loss has been observed. ${ }^{12}$ Several studies have also reported an accelerated progression to corpus atrophic gastritis during acid suppressive maintenance treatment with either $\mathrm{H}_{2}$ receptor antagonists or proton pump inhibitors (PPIs) in the presence of $\mathrm{H}$ pylori. ${ }^{13-17}$ The clinical consequences of this phenomenon are not yet fully understood. As a large number of patients with gastro-oesophageal reflux disease (GERD) are treated with acid suppressive maintenance therapy for many years, eradication of $\mathrm{H}$ pylor $i$ in these patients may be advisable to prevent these observed changes. Thus far however, it is not known if such a strategy would prevent the increased corpus gastritis and development of atrophy associated with profound acid suppressive therapy in $H$ pylori positive subjects. Therefore, the objective of this study was to determine if eradication of $H$ pylori prevents the histological changes induced by acid inhibition in the antral and corpus mucosa.

Abbreviations used in this paper: GERD, gastro-oesophageal reflux disease; PPI, proton pump inhibitors. 


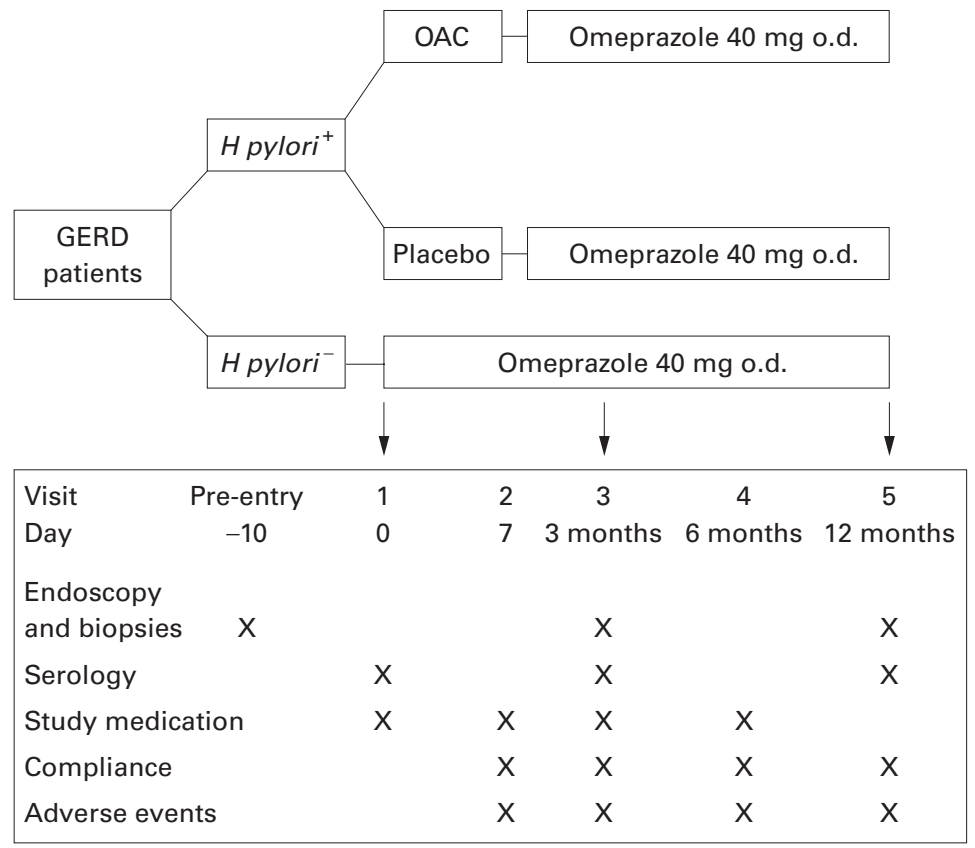

Figure 1 Study algorithm. Arrows indicate time schedule for endoscopy. O.d., once daily

\section{Patients and methods}

In a prospective, randomised, multicentre, case control study, patients referred with symptoms suggestive of GERD underwent flexible endoscopy of the upper gastrointestinal tract. Patients were eligible for entry into the study if endoscopic signs of reflux oesophagitis (grades 1-4 according to the Savary-Miller classification) were present. Exclusion criteria were prior or existing peptic ulcer disease, concurrent disease or therapy which could complicate the evaluation of the study such as known gastrointestinal disorders which might impair drug absorption, known liver or kidney disease, endocrine disease, suspected or confirmed malignancy, use of cytotoxic drugs, previous major oesophageal or gastric surgery, chronic alcoholism, penicillin allergy, drug abuse, and suspected poor compliance. The use of bismuth, antibiotics, or PPIs was not allowed one month before study entry.

The study was performed in accordance with the principles stated in the Declaration of Helsinki and the ethics committee of each participating hospital approved the study. Patients were included after written informed consent was obtained.

Table 1 Patient characteristics of those included in the final analysis, stratified by H pylori status at baseline and follow up. No differences in age, duration of GERD, or severity of reflux disease between groups

\begin{tabular}{llll}
\hline & H pylori $+/+^{a}$ & H pylori $+/-^{b}$ & H pylori $-/-^{c}$ \\
\hline No of patients & 24 & 33 & 26 \\
Age (years) (mean (range)) & $65(32-72)$ & $58(28-73)$ & $48(28-69)$ \\
Sex (M/F) & $14 / 10$ & $19 / 14$ & $12 / 14$ \\
Duration of GERD $^{\mathrm{d}}$ & $2 / 8 / 14$ & $1 / 16 / 16$ & $1 / 15 / 10$ \\
Reflux oesophagitis score $^{\mathrm{e}}$ & $14 / 10 / 0 / 0$ & $14 / 14 / 5 / 0$ & $18 / 7 / 1 / 0$
\end{tabular}

${ }^{\mathrm{a}} \mathrm{H}$ pylori positive during whole study period; ${ }^{\mathrm{b}} \mathrm{H}$ pylori positive at entry, negative at study end; ${ }^{\mathrm{c}} \mathrm{H}$ pylori negative controls.

${ }^{\mathrm{d}}$ No of patients with $<1 / 1-5 />5$ years of symptoms.

${ }^{\mathrm{N}} \mathrm{No}$ of patients with Savary-Miller score of $1 / 2 / 3 / 4$

GERD, gastro-oesophageal reflux disease.
CLINICAL AND LABORATORY ASSESSMENTS Patients were studied by upper gastrointestinal endoscopy at the pre-entry visit and after three and 12 months of follow up. Past and present medical history, GERD symptoms, and drug use were recorded at baseline and a physical examination was performed. At each endoscopy, four corpus and three antrum biopsy specimens were obtained with standard biopsy forceps for histopathological examination. In addition, one antrum and one corpus biopsy specimen were processed according to standard procedures for $H$ pylori culture. At the inclusion endoscopy, a CLO test (Delta West Ltd, Bentley, Western Australia) was also performed using one antrum and one corpus biopsy.

After haematoxylin-eosin and modified Giemsa staining, two experienced gastrointestinal pathologists, who were blinded to the clinical data and time of sampling, scored the specimens according to the updated Sydney classification. ${ }^{18}$ Separate scores were given for the presence of $H$ pylori ( 0 , no bacteria; 1 , focally few bacteria; 2 , more bacteria in several areas; 3 , abundance of bacteria in most glands), active (polynuclear leucocyte density: none/ mild/moderate/severe) and chronic inflammation (mononuclear cell infiltrate: normal/mild/ moderate/severe), atrophy, and intestinal metaplasia (normal/mild/moderate/marked). ${ }^{18}$ Special attention was given to differentiating between loss of appearance of glands due to inflammation and genuine atrophy with gland loss. In the event of discrepant results, the specimen was discussed until agreement was reached. In addition, serum was obtained at study entry and after three and 12 months for determination of $H$ pylori IgG antibodies using an IgG ELISA (Pyloriset EAI-G update, Orion Diagnostica, Espoo, Finland).

\section{RANDOMISATION PROCEDURE}

At baseline a patient was regarded as $H$ pylori positive if culture was positive or if both histology and the CLO test were positive. $H$ pylori positive patients were randomised within two weeks after the initial endoscopy to treatment with omeprazole $20 \mathrm{mg}$ twice daily, amoxicillin $1 \mathrm{~g}$ twice daily, and clarithromycin $500 \mathrm{mg}$ twice daily (OAC) for one week, followed by omeprazole $40 \mathrm{mg}$ once daily for 12 months, or to omeprazole $20 \mathrm{mg}$ twice daily with placebo amoxicillin and clarithromycin for one week followed by omeprazole $40 \mathrm{mg}$ once daily for one year. All $H$ pylori positive patients were seen within three days after termination of the triple therapy to check for compliance and side effects. Treatment in $H$ pylori negative patients consisted of omeprazole $40 \mathrm{mg}$ once daily for 12 months (fig 1 ).

All patients were seen for follow up visits after three, six, and 12 months. During follow up a patient was regarded as $H$ pylori positive if both culture and histology were positive. If culture and histology were contradictory, $H$ pylori serology was decisive. In this case the patient was considered to have become $H$ pylori negative if the $H$ pylori antibody titre of the 12 months' follow up serum had decreased to less 
Table 2 Median Sydney scores (0-3) for the antrum and corpus biopsy specimens at baseline (TO) and at 12 months'follow-up (T12) in persistently H pylori positive patients

\begin{tabular}{llll}
\hline & TO & T12 & p Value \\
\hline Antrum & & & \\
$\quad$ Chronic inflammation & 2 & 1 & $<0.0001$ \\
Active inflammation & 1 & 0 & 0.002 \\
Atrophy & 2 & 2 & 0.89 \\
Intestinal metaplasia & 0 & 0 & - \\
$\quad$ H pylori density & 3 & 1 & $<0.0001$ \\
Corpus & & & \\
Chronic inflammation & 1 & 2 & 0.03 \\
Active inflammation & 1 & 2 & 0.03 \\
Atrophy & 1 & 1 & 0.36 \\
Intestinal metaplasia & 0 & 0 & - \\
H pylori density & 2 & 1 & 0.01 \\
\hline
\end{tabular}

*Wilcoxon two sample test.

than $50 \%$ of baseline values. Intake of study medication, compliance, and adverse events were recorded at each visit.

STATISTICAL ANALYSIS

Pairwise comparisons of the three groups $(H$ pylori positive patients throughout the study, $H$ pylori positive patients who became $H$ pylori
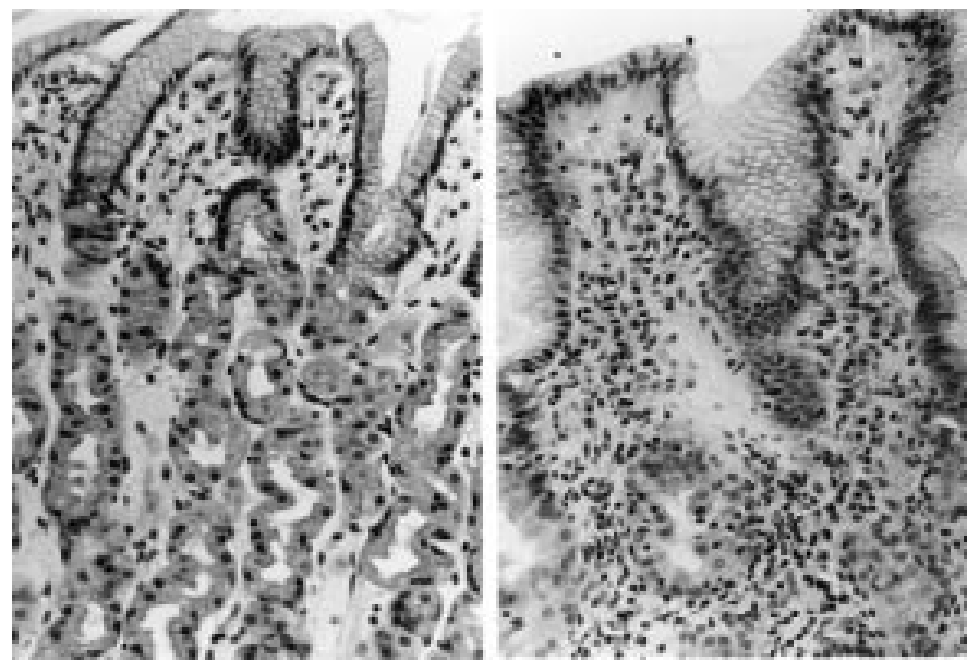

Figure 2 Corpus biopsy specimens of a persistently H pylori infected patient before (left) and after 12 months (right) of omeprazole treatment. Superficial gastritis (left) changes to more profound invasive, active, and chronic inflammation in the complete glandular layer.
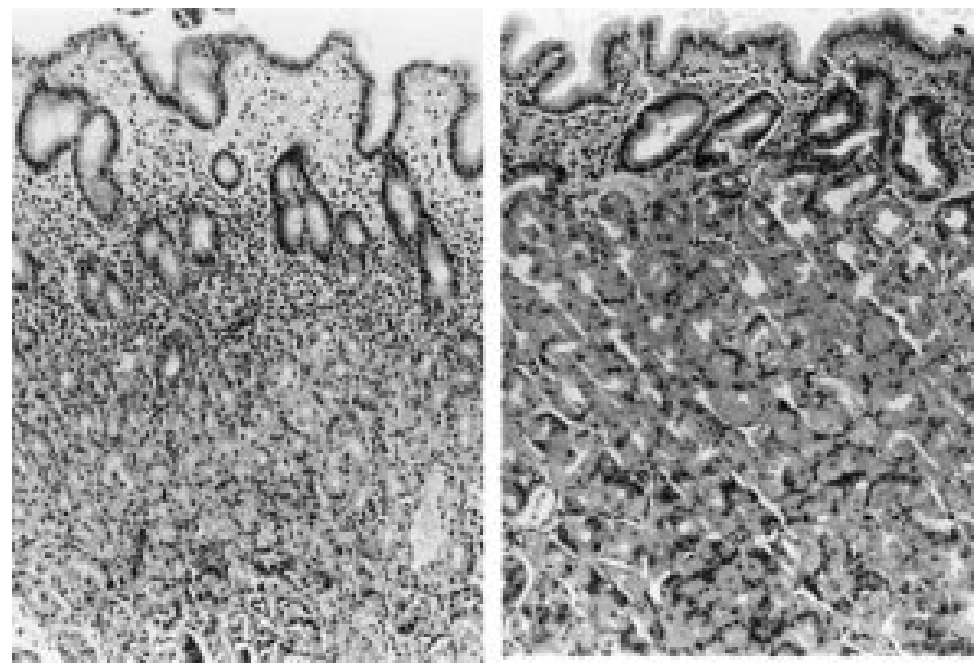

Figure 3 Corpus biopsy specimens of a successfully H pylori eradicated patient before (left) and after 12 months (right) of omeprazole treatment. The superficial inflammation at baseline (left) regresses during PPI treatment.
Table 3 Median Sydney scores (0-3) for the antrum and corpus biopsy specimens at baseline (TO) and at 12 months'follow-up (T12) for patients who became H pylori negative as a result of treatment

\begin{tabular}{llll}
\hline & To & T12 & $p$ Value \\
\hline Antrum & & & \\
$\quad$ Chronic inflammation & 2 & 1 & $<0.0001$ \\
Active inflammation & 1 & 0 & $<0.0001$ \\
Atrophy & 3 & 2 & 0.0009 \\
Intestinal metaplasia & 0 & 0 & - \\
$\quad$ H pylori density & 3 & 0 & $<0.0001$ \\
Corpus & & & \\
$\quad$ Chronic inflammation & 2 & 1 & 0.0001 \\
Active inflammation & 1 & 0 & $<0.0001$ \\
Atrophy & 1 & 1 & 0.12 \\
$\quad$ Intestinal metaplasia & 0 & 0 & - \\
$\quad$ H pylori density & 2 & 0 & $<0.0001$ \\
\hline ॠWilcoxon two sample test. & & &
\end{tabular}

negative as a result of treatment, and $H$ pylori negative controls) were performed at baseline and after 12 months of treatment for median Sydney scores for active and chronic inflammation, $H$ pylori colonisation density, atrophy, and intestinal metaplasia. For each patient the change in histopathological score during follow up was obtained by subtraction of baseline values from one year follow up values. Differences between groups were analysed non-parametrically using Wilcoxon two sample tests. Descriptive statistics are presented as median and interquartile ranges $(25-75 \%)$. In addition, histological scores at follow up were compared for patients who became $H$ pylori negative as a result of treatment and $H$ pylori negative control patients using an unpaired Mann-Whitney U test. A p value $<0.05$ was considered significant.

\section{Results}

A total of 100 patients were included; 17 were excluded from the final analysis because of incorrect stratification in four patients and loss to histological follow up of 13 patients. Fifty seven $(69 \%)$ of the remaining 83 patients were colonised with $H$ pylori. During the study, 24 patients remained $H$ pylori positive (of which 20 received placebo and four eradication treatment), whereas 33 were $H$ pylori positive at baseline and became negative as a result of treatment (10 received placebo and 23 antibiotic treatment). This was equivalent to an eradication rate of $85 \%$ for the OAC combination. The remaining 26 patients were $H$ pylori negative from the start of the study. The three groups did not differ in age, duration of GERD symptoms, or severity of reflux disease, as scored during the initial endoscopy (table 1).

INTRAGROUP COMPARISON

In the persistently $H$ pylori positive group, omeprazole therapy was associated with a decrease in the scores for the antrum for active and chronic inflammation and for $H$ pylori density $(\mathrm{p}<0.0001,0.0021$, and $<0.0001$, respectively). In contrast, active and chronic inflammation increased in the corpus (both $\mathrm{p}=0.03)$ despite a decrease in $H$ pylori density $(\mathrm{p}=0.012)$. Atrophy and intestinal metaplasia remained unchanged in both regions of the stomach (table 2; fig 2). In patients who became $H$ pylori negative as a result of 
Table 4 Comparison of median changes (T12-T0) in Sydney scores for persistently H pylori positive patients (Hp+/+; $n=24)$ and patients who became negative during treatment $(\mathrm{Hp}+1-; n=33)$. A significant decrease in active and chronic inflammation in the corpus was observed in the eradicated group

\begin{tabular}{|c|c|c|c|c|c|c|}
\hline & \multicolumn{3}{|l|}{ Antrum } & \multicolumn{3}{|l|}{ Corpus } \\
\hline & $\mathrm{Hp}^{+/+}$ & $H p+/-$ & $p$ Value & $H_{p}+/+$ & $H p+/-$ & $p$ Value \\
\hline Chronic inflammation ${ }^{\star}$ & -1 & -1 & 0.167 & 1 & -1 & 0.0001 \\
\hline Active inflammation $\star$ & -1 & -1 & 0.220 & 1 & -1 & 0.0001 \\
\hline Atrophy & 0 & -1 & 0.060 & 0 & 0 & 0.11 \\
\hline Metaplasia & 0 & 0 & - & 0 & 0 & - \\
\hline H pylori density & -2 & -3 & 0.0001 & -1 & -2 & 0.0001 \\
\hline
\end{tabular}

^Sydney score T12-T0.

treatment, active and chronic inflammation decreased in both the antrum and corpus (both $\mathrm{p} \leqslant 0.0001$ ) (table 3; fig 3).

The antrum specimens showed a decrease in atrophy score $(p=0.0009)$; median scores for metaplasia did not change. In the $H$ pylori negative control patients there were no changes in antrum or corpus biopsy specimens at follow up (data not shown).

INTERGROUP COMPARISON

Comparison of the histological changes in the persistently $H$ pylori positive patients and in those who became $H$ pylori negative as a result of treatment revealed the following. Patients who became $H$ pylori negative had a significant decrease in active and chronic inflammation in the corpus whereas persistently $H$ pylori positive patients showed a significant increase in chronic corpus inflammation. This contrast led to significant differences between groups for active and chronic inflammation in the corpus at 12 months (table 4). Antral active and chronic inflammation decreased in both groups; these changes did not differ signifi-

Chronic inflammation antrum

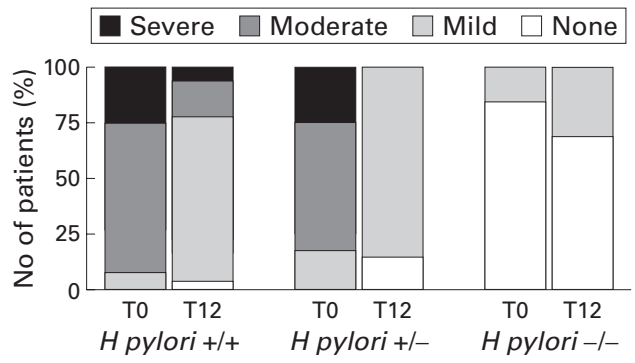

Chronic inflammation corpus

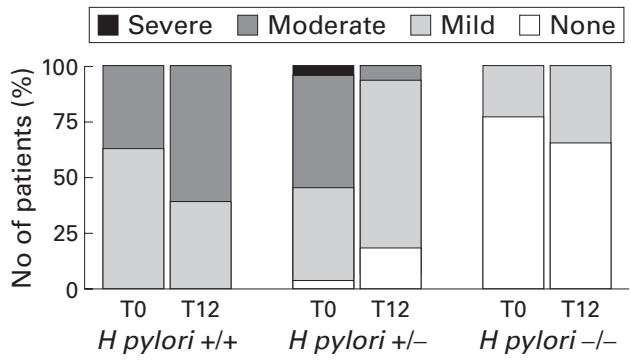

Figure 4 Changes in chronic inflammation parameters for the antrum and corpus at baseline (TO) and at follow up (T12) in persistently positive $H$ pylori patients (+/+), in patients who became $H$ pylori negative as a result of treatment (+/-), and in persistently negative controls $(-/-)$. Antrum biopsy specimens showed decreased chronic inflammation for $H$ pylori $+/+$ and $H$ pylori $+/-$. Chronic corpus inflammation improved in eradicated patients (+/-) whereas in $\mathrm{H}$ pylori $+/+$ patients inflammation increased during omeprazole treatment. cantly between groups (table 4). There was a trend for a significant difference between groups for changes in antral atrophy scores $(\mathrm{p}=0.06)$ but the median score in both groups was the same at the end of follow up and the eradicated group started with more severe antral atrophy at baseline. In addition, the eradicated group showed a difference in $H$ pylori density at the end of follow up compared with the persistent $H$ pylori positive patients (table 4). There were no differences between groups for other histological parameters for the antrum and corpus specimens (table 4).

At the 12 month follow up, median inflammation scores for the antrum and corpus for the successfully eradicated patients still differed from those who were $H$ pylori negative throughout the study $(\mathrm{p}=0.0004$ and 0.011 , respectively). No differences in other parameters were observed between those who were $H$ pylori negative at baseline and those who became negative as a result of treatment.

Changes in Sydney scores for inflammation and atrophy for the different groups during follow up are shown in figs 4 and 5 .

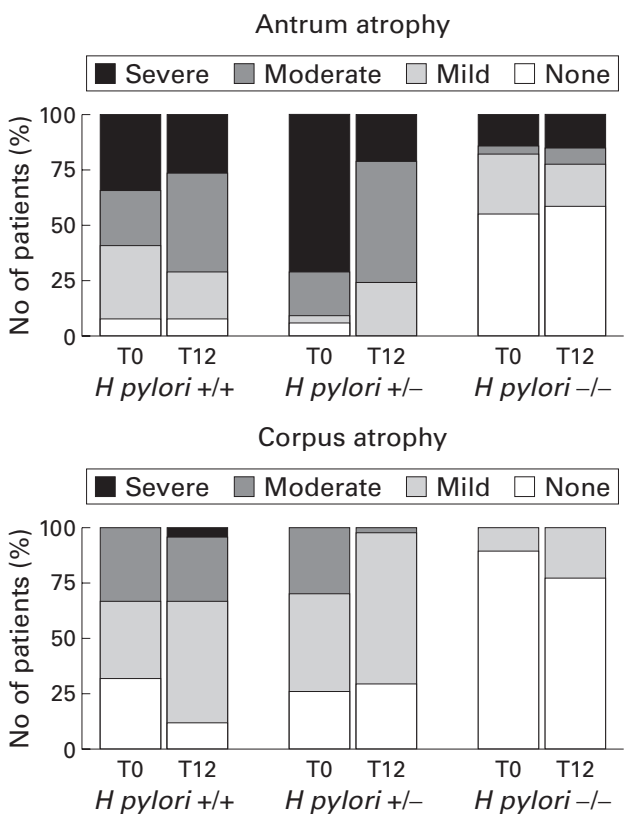

Figure 5 Changes in atrophy parameters for the antrum and corpus at baseline (TO) and at follow up (T12) in the persistently positive $H$ pylori patients $(+/+)$, in patients who became $H$ pylori negative as a result of treatment $(+-)$, and in persistently negative controls (-/-). Atrophy decreased in the antrum of $\mathrm{H}$ pylori+/- patients only (table 3). There were no changes in corpus atropy after one year of follow up. 
Discussion

$H$ pylori infection causes chronic mucosal inflammation which persists forever and may lead to atrophy and intestinal metaplasia. ${ }^{5} 61920$ These $H$ pylori induced conditions are associated with an increased risk for the development of gastric cancer and $H$ pylori is thus recognised as a class I carcinogen. ${ }^{8}$ The risk of development of gastric cancer depends on the distribution and severity of gastritis. The factors which influence the rate to progression of gland loss, metaplasia, and dysplasia are largely unknown but are thought to be related to host and environmental factors, and also to differences in the virulence of $H$ pylori. For example, $\operatorname{cag} A+$ strains are recognised as inducing atrophic gastritis and gastric cancer more rapidly than cag $A$-strains. ${ }^{21}{ }^{22}$ As for host factors, it appears that the severity and distribution of gastritis depends on host acid production. ${ }^{23}$ Treatment with a profound acid suppressor is associated with increased corpus inflammation. Based on these observations, the question was raised whether the increased corpus mucosal inflammation during profound acid suppressive maintenance therapy could increase the rate of progression to gland loss and atrophy.

Several groups have addressed this question. It appeared that the development of atrophic gastritis during acid suppressive maintenance therapy was strongly associated with the presence of $H$ pylori, and also that the rate of progression to atrophy was high in comparison with cohort studies in populations not using acid suppression. Accelerated progression to atrophy and argyrophil hyperplasia was observed during both short and long term treatment with acid suppressive drugs. ${ }^{15-17} 2425$ In $H$ pylori positive subjects the annual increase in corpus atrophy was consistently higher compared with $H$ pylori negative patients, ranging from 3 to $15 \%$, whereas in $H$ pylori negatives the increase in atrophy ranged from 1 to $3 \%{ }^{16}{ }^{17}$ The increased prevalence of corpus atrophy during acid suppressive therapy was not confirmed in another study ${ }^{26}$ in which the occurrence of corpus glandular atrophy was compared in GERD patients treated with either omeprazole or a Nissen fundoplication. Although the authors concluded that there was no difference in the development of atrophy in both groups after three years of follow up, the study was criticised on several points. All patients were initially treated with omeprazole for up to eight months, and some patients in the operated group also underwent vagotomy or were treated with omeprazole postoperatively because of persistent reflux symptoms. These factors affect acid production and thus could have biased the study results.

Most of the above studies made use of the updated Sydney classification for scoring the gastric biopsy specimens. This system has been well accepted for grading of gastric inflammation, atrophy, and metaplasia. A recent criticism on the observations of increased atrophy development suggested that the changes described did not represent genuine atrophy. ${ }^{27} \mathrm{It}$ was suggested that increased corpus inflamma- tion causes an influx of inflammatory cells by which these gastric glands become merely separated and pushed aside, without actual gland loss. However, we recently observed that in patients treated with omeprazole therapy for GERD, serum cobalamin levels decreased significantly only in those patients who were diagnosed as having had developed corpus atrophy. ${ }^{28}$ In this study the definition of atrophy was similar to that used in our previous publications. ${ }^{1323}$ This finding further supports the concept that "true" atrophy occurs during acid suppressive therapy, leading to physiological changes, and that the definition currently used is adequate. In addition, quantitative studies measuring the number of specialised glands in patients with different grades of atrophy confirmed a correlation between qualitative Sydney scores for atrophy and gland numbers. ${ }^{29}$ In the present study, $H$ pylori positive patients showed a decrease in inflammatory parameters and a reduction in $H$ pylori density in the antrum during omeprazole therapy. At the same time, a pronounced increase in corpus inflammation was found despite a decrease in $H$ pylori density in the corpus. These observations have been reported previously, both by us ${ }^{13} 23$ and by others. ${ }^{15-17} 2425$ The hypothesis is that they are related to the optimal growth conditions for $H$ pylori with changes in intragastric $\mathrm{pH}$ thus affecting the colonisation pattern. ${ }^{30}$ The observed increase in corpus inflammation despite no increase in bacterial numbers is thought to be related to closer contact of the bacteria with the corpus mucosa. In addition, autoimmune mechanisms, mediated by anti- $H$ pylori antibodies, have been suggested to play a role in corpus gastritis. ${ }^{31}$ However, we did not study this phenomenon in our study and thus cannot confirm if it occurred in our population.

Because of these changes in gastritis pattern and increased progression to gland loss, we recently suggested considering $H$ pylori eradication in patients on long term treatment with profound acid suppressive therapy. Thus far however, no study has investigated if such a strategy can prevent the reported histological changes in patients requiring long term PPI treatment. In our study the histopathological scores for active and chronic corpus inflammation improved significantly during omeprazole treatment in $H$ pylori eradicated patients compared with persistently $H$ pylori positive patients. In addition, in both $H$ pylori eradicated and persistently infected patients, omeprazole treatment for one year resulted in a decrease in active and chronic inflammation and $H$ pylori density of the antrum. These changes were not significantly different between the two groups. As expected, no changes were observed for any of the histological scores in the $H$ pylori negative control patients treated with omeprazole. Although an increase in body gastritis was observed in the $H$ pylori positive patients during omeprazole therapy, no significant changes in corpus atrophy scores were observed during follow up. This was probably because of the limited number of patients in our study and the relative short follow up 
period of 12 months. Similar findings were recently published by other investigators for lansoprazole. ${ }^{24}$ The efficacy of our one week eradication triple therapy was $85 \%$, which is in agreement with other data. ${ }^{32}$ Ten of 33 patients (30\%) defined as $H$ pylori negative at the end of the study were treated with omeprazole only. Acid suppressive therapy is known to have an inhibitory effect on $H$ pylori. Treatment with lansoprazole for one year for example, resulted in $H$ pylori "clearance" in $18 \%$ of patients. ${ }^{16}$ Although other studies found similar results, this loss of $H$ pylori is not in agreement with our previous experience during acid suppressive therapy. ${ }^{13}{ }^{23}$ This discrepancy may have been a result of our strict preset criteria of $H$ pylori positivity. We therefore analysed these 10 patients separately. It appeared that at the end of follow up, one patient had a positive culture but negative histology and a $60 \%$ decrease in serology titre. At the same visit, the other nine patients had negative histology and culture results. Six of these nine patients also had $>50 \%$ decrease in serology titre; in the remaining three, serology did not decrease $(n=2)$ or was unavailable $(n=1)$. The biopsy specimens of these three patients were therefore additionally analysed by $H$ pylori urease, vac $A$, and $\operatorname{cag} A$ PCR and tested negative. Therefore, we conclude that these 10 patients became $H$ pylori negative during treatment with omeprazole only.

The observed histological changes in gastritis and atrophy development are not unique to omeprazole and appear to occur with other PPIs, and with high dose $\mathrm{H}_{2}$ receptors antagonists. ${ }^{1014162333}$ In our study, with follow up after one year, we observed a significant increase in corpus gastritis in persistently $H$ pylori infected patients only. This is the first prospective study demonstrating that $H$ pylori eradication can prevent the occurrence of these changes, suggesting the pivotal role of acid suppressive therapy in the development of these alterations. This supports our hypothesis that long term acid suppressive therapy for GERD together with $H$ pylor $i$ is associated with increased corpus inflammation. Whether the observed mucosal changes eventually leads to an increased risk of gastric cancer during acid suppressive therapy is speculative. The development of atrophic gastritis is not the only factor and it has been suggested that a predominantly corpus gastritis pattern with increased mucosal proliferation increases the risk of the diffuse type of gastric cancer in particular. ${ }^{34}$ This hypothesis is based on studies in patients with early diffuse type gastric cancer and their relatives who appeared to often have a predominantly corpus gastritis without signs of gland loss or metaplasia. Studies over longer periods may be necessary to resolve these issues. Additional attention has to be given to the decreased inflammation of the antrum during acid suppression. In theory, this decrease could also reduce the risk of cancer of the distal stomach. Based on the above mentioned data and on the fact that $H$ pylori can be eradicated easily, it has been advised to consider eradication of $H$ pylori during omeprazole maintenance treatment. ${ }^{13} 35$ Whether or not such a strategy is beneficial and devoid of effect on the control of GERD symptoms needs to be demonstrated in further randomised intervention studies.

In conclusion, $H$ pylori eradication at the start of proton pump inhibitor therapy prevented the increase in corpus gastritis associated with such therapy in $H$ pylori positive patients. This was associated with a decrease in antral gastritis activity. Longer follow up is needed to study if $H$ pylori eradication as such may prevent the development of atrophic gastritis.

1 Marshall BJ, Armstrong JA, McGechie DB, et al. Attempt to fulfil Koch's postulates for pyloric Campylobacter. Med $\mathcal{F}$
Austr 1985;142:436-9.

2 Morris A, Nicholson G. Ingestion of Campylobacter pyloridis causes gastritis and raised fasting gastric pH. Am f Gastroenterol 1987;82:192-9.

3 Dixon MF. Helicobacter pylori and peptic ulceration; histopathological aspects. $\mathcal{f}$ Gastroenterol Hepatol 1991;6: 125-30.

4 Rauws EAJ, Langenberg W, Houthoff HJ, et al. Campylobacter pyloridis-associated chronic active antral gastritis. A prospective study of its prevalence and the effects of antibacterial and anti-ulcer treatment. Gastroenterology 1988;94:33-40.

5 Sipponen P. Long-term consequences of gastroduodenal inflammation. Eur f Gastroenterol Hepatol 1992;4 (suppl 2):S25-9.

6 Kuipers EJ, Uyterlinde AM, Peña AS, et al. Long term sequelae of Helicobacter pylori gastritis. Lancet 1995;345: 1525-8.

7 Parsonnet J, Friedman GD, Vandersteen DP, et al. Helicobacter pylori infection and the risk of gastric Helicobacter pylori infection and the risk
carcinoma. N Engl f Med 1991;325:1127-31.

carcinoma. $N$ Engl f Med $1991 ; 325: 1127-31$.
8 IARC monographs on the evaluation of carcinogenic risks to IARC monographs on the evaluation of carcinogenic risks to
humans, vol 61, schistosomes, liver flukes and Helicobacter pylori. Lyon: International Agency for Research on Cancer, 1994

9 Miehlke S, Hackelsberger A, Meining A, et al. Histological diagnosis of Helicobacter pylori gastritis is predictive of a high risk of gastric carcinoma. Int $\mathcal{F}$ Cancer 1997;73:837-9.

10 Logan RPH, Walker MM, Misiewicz JJ, et al. Changes in the intragastric distribution of Helicobacter pylori during treatment with omeprazole. Gut 1995;36:12-16.

11 Sachs G. Gastritis, Helicobacter pylori, and proton pump inhibitors. Gastroenterology 1997;112:1033-6.

12 Kuipers EJ, Lee A, Klinkenberg-Knol EC, Meuwissen SGM. Review article: The development of atrophic gastritis-Helicobacter pylori and the effects of acid suppresgastritis-Helicobacter pylori and the effects of acid suppr
sive therapy. Aliment Pharmacol Ther 1995;9:331-40.

13 Kuipers EJ, Lundell L, Klinkenberg-Knol EC, et al. Atrophic gastritis and Helicobacter pylori infection in patients with reflux oesophagitis treated with omeprazole or fundoplication. N Engl F Med 1996;334:1018-22.

14 Kolkman JJ, Hazenberg HJA, Dekker N, et al. The effect of acid suppression on H. pylori gastritis is dose dependant. Gastroenterology 1998;112:A128.

15 Lamberts R, Creutzfeldt W, Strüber HG, et al. Long-term omeprazole therapy in peptic ulcer disease: gastrin, endocrine cell growth, and gastritis. Gastroenterology 1993; 104:1356-70.

16 Eissele R, Brunner G, Simon B, et al. Gastric mucosa during treatment with lansoprazole: Helicobacter pylori is a risk factor for argyrophil-cell hyperplasia. Gastroenterology 1997; 112:707-17.

17 Berstad AE, Hatlebakk JG, Maartmann-Moe H, et al. Helicobacter pylori gastritis and epithelial cell proliferation in patients with reflux oesophagitis after treatment with patients with reflux oesophagiti
lansoprazole. Gut 1997;41:740-7.

18 Dixon MF, Genta RM, Yardley JH, et al. Classification and grading of gastritis. The updated Sydney system. Am $\mathcal{F}$ Surg Pathol 1996;20:1161-81.

19 Sipponen P, Kekki M, Haapakoski J, et al. Gastric cancer risk in chronic atrophic gastritis: statistical calculations of risk in chronic atrophic gastritis: statistical calculational data. Int $\mathcal{F}$ Cancer 1985;35:173-7.

20 Correa P. Human gastric carcinogenesis: a multistep and multifactorial process. Cancer Res 1992;52:6735-40

21 Kuipers EJ, Pérez-Pérez GI, Meuwissen SGM, et al. Helicobacter pylori and atrophic gastritis; importance of the cag $\mathrm{A}$ status. $\mathcal{F}$ Natl Cancer Inst 1995;87:1777-80

22 Blaser MJ, Pérez-Pérez GI, Kleanthous $\mathrm{H}$, et al. Infection with Helicobacter pylori strains possessing cag $A$ is associated with an increased risk of developing adenocarcinoma of the stomach. Cancer Res 1995;55:2111-15.

23 Kuipers EJ, Uyterlinde AM, Peña AS, et al. Increase of Helicobacter pylori associated corpus gastritis during acid suppressive therapy: Implications for long-term safety. $\mathrm{Am}$ f Gastroenterol 1995;90:1401-6.

24 Stolte M, Meining A, Schmitz JM, et al. Changes in Helicobacter pylori-induced gastritis in the antrum and corpus during 12 months of treatment with omeprazole and lansoprazole in patients with gastro-oesophageal reflux disease. prazole in patients with gastro-oesophageal
Aliment Pharmacol Ther 1998;12:247-53. 
25 Meining A, Bossecker H, Caspary WF, et al. H2-receptor antagonists and antacids have an aggravating effect on Helicobacter pylori gastritis in duodenal ulcer patients.

26 Lundell L, Miettinen P, Myrvold HE, et al. Lack of effect of acid suppressive therapy on gastric atrophy. Gastroenterology 1999;117:319-26.

27 Genta RM. Acid suppression and gastric atrophy: sifting fact from fiction. Gut 1998;43 (suppl 1):S35-8.

28 Schenk BE, Kuipers EJ, Klinkenberg-Knol EC, et al. Atrophic gastritis during long-term omeprazole therapy affects serum vitamin $\mathrm{B}_{12}$ levels. Aliment Pharmacol Ther 1999;13:1343-6.

29 Grieken van NCT, Weiss MM, Meyer GA, et al. Rapid quantitative assessment of gastric corpus atrophy in tissue sections. Gastroenterology 1999;4:A177.

30 Sachs, G. Gastritis, Helicobacter pylori, and proton pump inhibitors. Gastroenterology 1997;112:1033-6.
31 Stolte M, Baumann K, Bethke B, et al. Active autoimmune gastritis without total atrophy of the glands. $Z$ Gastroenterol gastritis without tota

32 Lind T, Veldhuysen van Zanten S, Unge P, et al. Eradication of Helicobacter pylori using one-week triple therapies combining omeprazole and two antimicrobials: The MACH 1 study. Helicobacter 1996;1:138-44

33 Athmann C, Mander I, Brunner G, et al. Histology and safety parameters during long-term maintenance with pantozole in severe acid-peptic disease. Gastroenterology 1998;114:A60.

34 Meining AG, Bayerdorffer E, Stolte M. Helicobacter pylori gastritis of the gastric cancer phenotype in relatives of gastric carcinoma patients. Eur 7 Gastroenterol Hepatol 1999;11:717-20.

35 Moayyedi P, Morrow S, Peacock R, et al. Changing patterns of H. pylori gastritis in long-standing acid suppression. Gastroenterology 1999;116:A257. 